\title{
Correction to: Determination of the protection period of fungicides used for control of Sclerotinia stem rot in soybean through bioassay and chromatography
}

\author{
Thomas J. J. Miorini 1 • Carlos Gilberto Raetano 1 \\ Matheus M. Negrisoli • Oscar Pérez-Hernández
}

Published online: 19 February 2021

(C) Koninklijke Nederlandse Planteziektenkundige Vereniging 2021

\section{Correction to: Eur J Plant Pathol}

https://doi.org/10.1007/s10658-021-02212-Z

This erratum is published as several typesetting errors introduced during proofing related with Tables 2,3,4,5 and has now been corrected.

Original article has been updated.

The online version of the original article can be found at https://doi.org/10.1007/s10658-021-02212-z

\section{T. J. J. Miorini}

Carrington Research Extension Center, North Dakota State

University, Carrington, ND 58421, USA

C. G. Raetano $(\varangle) \cdot$ M. M. Negrisoli

Department of Plant Protection, School of Agriculture, São Paulo State University (UNESP), Botucatu, SP 18610-034, Brazil e-mail: carlos.raetano@unesp.br

O. Pérez-Hernández

School of Agricultural Sciences, Northwest Missouri State University, Maryville, MO 64468, USA 